\title{
Source par décharge capillaire pour la lithographie EUV
}

\author{
C. Cachoncinlle, E. Robert, O. Sarroukh, T. Gonthiez, R. Viladrosa, \\ C. Fleurier et J.M. Pouvesle
}

\author{
GREMI ESPEO, Université d'Orléans, 14 rue d'Issoudun, BP. 6744, 45067 Orléans cedex 02, \\ France
}

\begin{abstract}
Résumé : Dans ce travail, nous présentons les caractéristiques de la lampe à décharge capillaire Capella. Cette source de rayonnement EUV peut produire un flux de photon supérieur au Watt $(2 \% \mathrm{BW}, 13.5 \mathrm{~nm})$ avec un rendement à la prise de courant de plus de $0.1 \%$. Elle fonctionne soit en mode pulsé à $150 \mathrm{~Hz}$ soit en rafale en générant des trains d'impulsion au kilohertz. La stabilité spatiale de la source est meilleure que $50 \mu m$ et sa stabilité temporelle atteint $0.3 \%$. La longévité de la source est supérieure à $10^{7}$ tirs.
\end{abstract}

\section{INTRODUCTION}

La course à l'intégration des composants semi-conducteurs élémentaires, commencée il y a plus de trente ans, conduit maintenant l'industrie de la microélectronique à user de tout son savoir-faire technologique pour faire reculer les limites des techniques de la lithographie optique. Aujourd'hui la réalisation des très petites structures est principalement limitée par la longueur d'onde des sources optiques commercialement disponibles. Après la génération des sources laser $\mathrm{KrF}$ à $248 \mathrm{~nm}$, les nouvelles chaînes de production commencent maintenant à s'équiper de sources laser ArF à 193 nm. Pour le futur immédiat, il est prévu que les sources laser $F_{2}$ à $157 \mathrm{~nm}$ prendront le relais dans les années 2005. Mais, ensuite, pour de plus forte intégration, il faudra utiliser des sources émettant en dessous de $100 \mathrm{~nm}$ ce qui soulève alors nombre de difficultés nouvelles.

Au-delà des puissants instruments de laboratoire qui produisent des radiations de courtes longueurs d'onde dans le domaine spectral de l'Extrême Ultraviolet (EUV), l'industrie de la microélectronique attend des sources, non nécessairement cohérentes, mais fiables, puissantes, d'un coût raisonnable et aisément intégrables sur les steppers des chaînes de production.

De nombreux verrous technologiques sont dorénavant « ouverts », et il semble fort probable que la réalisation de circuits intégrés de dimension inférieure à $50 \mathrm{~nm}$ au moyen de sources optiques émettant à $13.5 \mathrm{~nm}$ va rapidement voir le jour. Cette nouvelle lithographie EUV est attendue en production pour les années 2008.

Dans le cadre du projet français PREUVE, nous avons étudié un concept de Lampe EUV pouvant répondre à quelques applications liées à cette nouvelle lithographie. En particulier, CAPELLA (Capillary Euv Lamp for Lithography Aproach) semble parfaitement adaptée à la métrologie à $13.5 \mathrm{~nm}$. Ce concept de lampe est basé sur la technique simple et fiable des décharges capillaires. Cette technique permet, de part son excellent rendement énergétique à la prise de courant, d'obtenir le fonctionnement à très haut taux de répétition, permettant de délivrer des puissances moyennes significatives

Nous présenterons ici les principales caractéristiques de CAPELLA. La puissance rayonnée dans $2 \%$ de bande passante autour de la longueur d'onde standard $13.5 \mathrm{~nm}$ en fait une source alternative très compétitive vis à vis d'instruments beaucoup plus imposant. La compacité et la simplicité de mise en œuvre ont été particulièrement pensées pour l'utilisateur. Le principe même des décharges capillaires en 
fait une source de grande stabilité spatiale. La reproductibilité tir à tir ainsi que les fluctuations de la puissance rayonnée sur des temps longs sont parmi ses caractéristiques les plus originales. Enfin l'extrême longévité de la source ainsi que son faible taux de pollution en font une véritable lampe spectrale utilisable pour diverses applications scientifiques et commerciales.

\section{RESULTATS EXPERIMENTAUX}

\subsection{Rendement énergétique}

La production de flux importants de photon de grande énergie, de l'ordre de la centaine d'électron-Volt, peut être aisément obtenue à partir de plasmas transitoires de forte température. En première approximation, la densité spectrale de rayonnement est gouvernée par la température du milieu. La loi du corps noir donne une bonne indication de l'ordre des températures à atteindre pour émettre le maximum de radiation dans l'EUV. Pour une longueur d'onde voisine de $13 \mathrm{~nm}$, la température correspondant au maximum d'émission, donné par le loi de Wien, est de quelques $20 \mathrm{eV}$.

Bien évidemment cette approximation grossière doit être corrigée pour tenir compte des transitions excitées et l'émissivité $\eta(\lambda)$ résultante est fortement dépendante de la longueur d'onde. Cette émissivité doit donc être écrite comme le produit de la loi de Wien, $W(\lambda)$, par un coefficient d'émission spectrale, $\mathrm{k}(\lambda)$. La loi de rayonnement du corps noir nous fournit une valeur maximale de la puissance qui peut être extraite du milieu. Pour les applications liées à la lithographie EUV, il est intéressant d'estimer le rendement maximum que l'on peut espérer obtenir dans une bande spectrale correspondant au maximum de réflectivité des miroirs en Mo-Si: soit une bande spectrale de $0.3 \mathrm{nn}$ centré à $13.5 \mathrm{nn}$. Pour un plasma de $20 \mathrm{eV}$ correspondant à la longueur d'onde optimale de $13.5 \mathrm{~nm}$, le rendement théorique maximal est de $1.5 \%$. Il paraît alors difficile d'espérer obtenir des sources de rendement à la prise de courant supérieur au dixième de cette valeur, soit quelques millièmes, après toutes les pertes encourues.

\subsection{Caractéristique du plasma}

La modélisation $\mathrm{MHD}$ d'une décharge capillaire remplie de xénon a été réalisée [1]. Les résultats permettent d'estimer la température et la densité électroniques atteintes dans nos conditions typiques de fonctionnement. Pour un courant de $2.6 \mathrm{kA}$, et une pression comprise entre 0.2 et $1 \mathrm{mbar}$, la température électronique prédite atteint une valeur comprise entre 40 et $20 \mathrm{eV}$ respectivement. Ces valeurs sont obtenues à l'instant de pinch soit 40 et 50 ns après l'amorçage de la décharge. Ces valeurs sont en bon accord avec la température du corps noir idéalement requis pour optimiser le rendement. Lors de la compression maximale du plasma, à ces mêmes instants, les densités électroniques peuvent atteindre des valeurs comprises entre $2.010^{17} \mathrm{~cm}^{-3}$ et $4.110^{17} \mathrm{~cm}^{-3}$ respectivement pour des pressions de 0.2 et $1 \mathrm{mbar}$. Notons que le courant reste trop modéré pour obtenir une compression importante du plasma. Le facteur de compression reste inférieur à 3 , conformément à ce qui est observé expérimentalement. Soulignons que, pour les applications d'insolation de résines photosensible, il est n'est pas avantageux de produire des sources de trop petites tailles qui limiteraient alors le champ d'exposition.

\subsection{Spectre}

Capella fonctionne sous flux de xénon à une pression de l'ordre du millibar. Le spectre de la décharge est caractéristique des plasmas de Xénon de forte densité tel que ceux obtenus dans des plasmas produits par laser. Les paramètres expérimentaux ont été ajustés pour optimiser la production de rayonnement à 13.5 nm. Les spectres [2] font apparaître des transitions du Xénon fortement ionisé attribuées au Xe VIII à Xe XI. Notons que les bases de données spectroscopiques font fortement défaut sur les états ioniques multichargés du Xénon. Néanmoins, une modélisation simple permet de corroborer certaines de nos observations expérimentales [1]. 


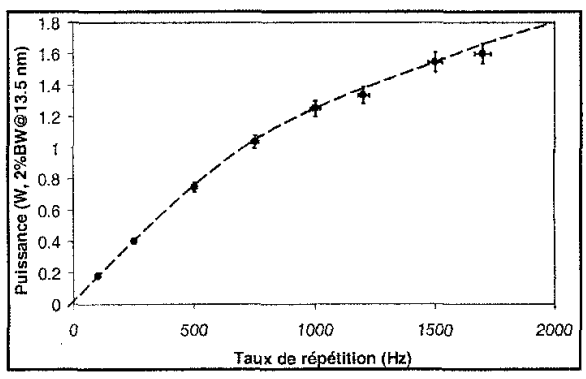

Figure 1: Puissance émise par Capella

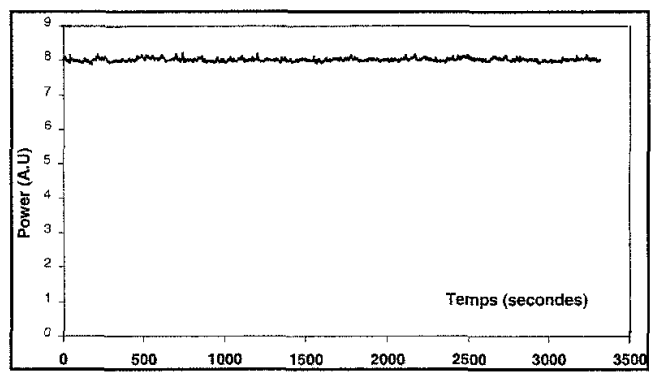

Figure 2: Stabilité temporelle de Capella $(100 \mathrm{~Hz})$

\subsection{Mode opératoire}

Bien évidement les températures nécessaires ne peuvent être maintenues que transitoirement. Capella délivre des impulsions de photons d'une durée de $50 \mathrm{~ns}$ (FWHM). Dans ces conditions, des températures de quelques centaines de milliers de Kelvin peuvent être atteintes même pour des énergies emmagasinées de l'ordre d'un Joule par tir. La puissance de la source est alors obtenue en augmentant le taux de répétition des tirs. Nous avons testé les performances de Capella jusqu'à $4 \mathrm{kHz}$. Les seules limites qui sont apparues sont liées à la limitation en puissance de l'alimentation électrique utilisée. Elle n'est pas capable de maintenir la charge des condensateurs au delà de $2 \mathrm{kHz}$ ce qui provoque un décrochement de la puissance EUV (cf Fig 1). Le plasma de Capella peut produire plus de $2 \mathrm{~W}$ de puissance dans les 0.3 nm de bande passante des miroirs MoSi ( soit 2\% BW à $13.5 \mathrm{~nm}$ ). Capella ayant été conçue pour être utilisée comme source d'illumination sur le BEL ( Banc d'Essai de Lithographie), l'angle solide de collection du rayonnement a été limité à $2 \%$ de $4 \pi$ stéradians en accord avec les autres composants optiques.

Bien que la source puisse opérer à très haut taux de répétition, elle a été conçue pour illuminer les résines photosensibles pendant le temps le plus court possible (pour limiter le problème de bougé pendant l'exposition). Sans refroidissement particulier, la source délivre des trains d'impulsion limités à 10 secondes pour limiter le flux thermique à évacuer. Nous l'avons cependant testé à faible taux de répétition $(150 \mathrm{~Hz})$ pendant plusieurs heures en continu avec un simple refroidissement à eau.

\subsection{Stabilité temporelle}

La stabilité temporelle de Capella a été mesurée dans les deux modes de fonctionnement décrit plus haut : A faible cadence $(100 \mathrm{~Hz}$ ) les fluctuations de puissance mesurées sur 1 heure n'excédent pas $0.6 \%$ (écart type sur 3600 échantillons). Pour la plus grande part, elles proviennent de variation pendant les premières minutes. En mode dit «rafale », les essais réalisés sur des trains d'impulsion au kilohertz espacés de quelques minutes prouvent que la dose de rayonnement produite est reproductible a plus de $0.3 \%$ près.

\subsection{Longévité}

Les tests de longévité de la source sont actuellement en cours. Nous avons déjà opéré quelques dizaines de million de tirs sans aucune défaillance. La technologie employée permet de minimiser l'ablation du tube capillaire. Il est probable que la durée de vie de la source sera fixée par le commutateur et le tube capillaire en alumine. Nous utilisons des commutateurs thyratrons éprouvés pour leur robustesse et leur fiabilité. Ils permettent en outre de synchroniser la source avec un autre appareil. 


\subsection{Stabilité spatiale}

Les mesures de stabilité spatiale de Capella ont été obtenues par imagerie sténopé filtrée en longueur d'onde. Nous avons utilisé un filtre $\mathrm{Zr}$ de $160 \mathrm{~nm}$ d'épaisseur. Les images du point source correspondent au domaine spectrale s'étendant de 10 à $20 \mathrm{~nm}$. L'utilisation d'une caméra ICCD couplé à un détecteur multicanal résolu temporellement a permis de suivre les phases de compression et d'extension du plasma. Les fluctuations spatiales ont été estimées à 50 micromètres. Cette bonne stabilité est due au confinement du plasma sur l'axe du capillaire lors de la phase de constriction magnétique. Il est probable que la durée de l'impulsion ne laisse guère le temps aux instabilités de se développer.

\subsection{Pollution des optiques}

Les décharges capillaires sont connues pour éjecter un grand nombre de fragments massiques : les débris. Ils proviennent généralement essentiellement du matériau du capillaire et de celui des électrodes. Nous avons aussi optimisé la décharge pour minimiser cette pollution. Les tests effectués [2] sur des échantillons en silicium cristallin placés devant la source révèlent des taux de dépôts de l'ordre de un Angström de matériau déposé par million de tirs effectués. Les observations de ces échantillons ne montrent pas d'impacts particuliers, il semble que les matériaux se déposent de façon homogène. Les analyses ont révélé, outre la présence des matériaux des électrodes, principalement du tungstène, la présence significative $(20 \%)$ de xénon profondément implanté dans le substrat. Ceci témoigne de la présence d'une population d'ions présentant une énergie cinétique importante. Des ions de xénon d'énergie cinétique voisine du keV ont déjà été observés dans les sources plasma créé par laser.

\section{CONClusion}

Nous avons présenté dans ce travail les résultats de nos études sur les potentialités d'une source par décharge capillaire pour la production de photons EUV principalement optimisée pour les applications de la lithographie. Nous avons montré que la source Capella, réalisée dans le cadre du projet français PREUVE, présente quelques caractéristiques remarquables. Le rendement énergétique atteint plus de $0.1 \%(2 \% \mathrm{BW}$ à $13.5 \mathrm{~nm})$. La température du plasma transitoire atteint 20 à $40 \mathrm{eV}$ selon la pression locale dans le capillaire. L'ajustement des paramètres de la décharge a été mené de façon à optimiser le spectre pour rayonner préférentiellement dans la bande passante des miroirs MoSi. La source Capella peut fonctionner soit en mode pulsé à des taux de répétition de $150 \mathrm{~Hz}$ pendant des heures, soit en rafales de durée inférieure à 10 secondes au kilohertz. Ce dernier mode étant spécifiquement développé pour le BEL. La stabilité temporelle $(0.3 \%)$ est particulièrement remarquable dans ce mode de fonctionnement. La longévité de la source est supérieure à 10 millions de tirs. Les fluctuations spatiales du point source restent inférieures à $50 \mu \mathrm{m}$. L'éjection de débris par la décharge reste modérée. Les taux de dépôt mesurés sont de l'ordre de $10^{-6} \AA ̊ \AA$ par tir.

\section{Remerciements}

Ce programme a bénéficié du soutien financier du ministère de l'industrie au travers du projet PREUVE. Nous tenons également à remercier le Pr. M. Vrbova de l'Université Technologique de Prague, ainsi que toute son équipe pour la collaboration fructueuse sur le travail de modélisation.

\section{Références}

[1] Cachoncinlle C., Robert E., Sarroukh O.,Gonthiez T., Viladrosa R., Fleurier C.,Pouvesle J.M., Vrba P., Vrbova M., Bobrova N.and Sasorov P." Dynamics and emission characteristics of xenon capillary discharge", 8th Int. Conf. on X-Ray Lasers ;Aspen U.S. 27-31 mai 2002

[2] Sarroukh O., Robert E., Gonthiez T., Thomann A.L., Viladrosa R., Fleurier C.,Pouvesle J.M., Cachoncinlle $\mathrm{C}$, Capella : une source de rayonnement extreme UV à $13.5 \mathrm{~nm}$ par décharge électrique », colloque UVX 2002, Oléron Fr, 11-14 juin 2002. 